\title{
Differential Expression of mRNAs in the Brain Tissues of Patients with Alzheimer's Disease Based on GEO Expression Profile and Its Clinical Significance
}

\author{
Guowei Ma $\mathbb{D}^{1,2}$ Mingyan Liu $\mathbb{D}^{1,2}$ Ke Du, ${ }^{1,2}$ Xin Zhong, ${ }^{1,2}$ \\ Shiqiang Gong, ${ }^{1,2}$ Linchi Jiao, ${ }^{1,2}$ and Minjie Wei $\mathbb{D}^{1,2}$ \\ ${ }^{1}$ School of Pharmacy, Department of Pharmacology, China Medical University, Shenyang, Liaoning, China \\ ${ }^{2}$ Liaoning Key Laboratory of Molecular Targeted Anti-Tumor Drug Development and Evaluation, Shenyang, Liaoning, China
}

Correspondence should be addressed to Mingyan Liu; saffer@163.com and Minjie Wei; minjie_wei@163.com

Received 12 September 2018; Revised 28 December 2018; Accepted 4 February 2019; Published 26 February 2019

Academic Editor: Xudong Huang

Copyright (C) 2019 Guowei Ma et al. This is an open access article distributed under the Creative Commons Attribution License, which permits unrestricted use, distribution, and reproduction in any medium, provided the original work is properly cited.

Background. Early diagnosis of Alzheimer's disease (AD) is an urgent point for AD prevention and treatment. The biomarkers of $\mathrm{AD}$ still remain indefinite. Based on the bioinformatics analysis of mRNA differential expressions in the brain tissues and the peripheral blood samples of Alzheimer's disease (AD) patients, we investigated the target mRNAs that could be used as an $\mathrm{AD}$ biomarker and developed a new effective, practical clinical examination program. Methods. We compared the AD peripheral blood mononuclear cells (PBMCs) expression dataset (GEO accession GSE4226 and GSE18309) with AD brain tissue expression datasets (GEO accessions GSE1297 and GSE5281) from GEO in the present study. The GEO gene database was used to download the appropriate gene expression profiles to analyze the differential mRNA expressions between brain tissue and blood of AD patients and normal elderly. The Venn diagram was used to screen out the differential expression of mRNAs between the brain tissue and blood. The protein-protein interaction network map (PPI) was used to view the correlation between the possible genes. GO (gene ontology) and KEGG (Kyoto Gene and Genomic Encyclopedia) were used for gene enrichment analysis to determine the major affected genes and the function or pathway. Results. Bioinformatics analysis revealed that there were differentially expressed genes in peripheral blood and hippocampus of AD patients. There were 4958 differential mRNAs in GSE18309, 577 differential mRNAs in GSE4226 in AD PBMCs sample, 7464 differential mRNAs in GSE5281, and 317 differential mRNAs in GSE129 in AD brain tissues, when comparing between AD patients and healthy elderly. Two mRNAs of RAB7A and ITGB1 coexpressed in hippocampus and peripheral blood were screened. Furthermore, functions of differential genes were enriched by the PPI network map, GO, and KEGG analysis, and finally the chemotaxis, adhesion, and inflammatory reactions were found out, respectively. Conclusions. ITGB1 and RAB7A mRNA expressions were both changed in hippocampus and PBMCs, highly suggested being used as an AD biomarker with $\mathrm{AD}$. Also, according to the results of this analysis, it is indicated that we can test the blood routine of the elderly for 2-3 years at a frequency of 6 months or one year. When a patient continuously detects the inflammatory manifestations, it is indicated as a potentially high-risk $\mathrm{AD}$ patient for $\mathrm{AD}$ prevention.

\section{Introduction}

$\mathrm{AD}$ is a kind of dementia that was first discovered and named by the German psychiatrist and neuropathologist Alzheimer Alois in 1906 [1]. AD is a chronic neurodegenerative disease from mild-to-severe symptoms with the main clinical manifestations such as memory loss, cognitive dysfunction, behavioral abnormalities, and social disorders [2]. In 2015, there were 46.8 million $\mathrm{AD}$ patients worldwide, and it is expected to reach 74.7 million in 2030 and more than 131.5 million in 2050. The global costs of AD care in 2015 totaled up to 818 billion dollars. In just three years, this amount will increase to 1 trillion dollars, and in 2030 it will reach 2 trillion dollars [3]. If the early detection and intervention are available and effective, the onset will be delayed for 5-10 years, which can exceedingly reduce the family burden and 
medical system pressure [4]. Therefore, the early diagnosis of Alzheimer's disease is extremely important.

Many hypotheses were proposed in the pathogenesis of $\mathrm{AD}$. As we know, the main pathogenesis of $\mathrm{AD}$ was the abnormal deposition of amyloid $\beta$-protein $(A \beta)$, hyperphosphorylation of Tau protein, oxidative stress, mitochondrial cascade, inflammatory response, and barrier to insulin signaling pathways. However, AD was a complex disease caused by genetic factors and environmental factors. A single hypothesis might not fully explain the full incidence of $\mathrm{AD}[5$, 6]. Current researches indicated that the main pathological feature of $\mathrm{AD}$ patients was that $\mathrm{A} \beta$ aggregated into senile plaques and abnormal accumulation of intracellular Tau protein to form the neurofibrillary tangles (NFT) and neuronal death [7]. The current mainstream detection methods were shown in Table 1 [8-11], but each method was not perfect. Therefore, in this study, we expected to explore more suitable detection methods through bioinformatics analysis. In recent years, bioinformatics prediction and computer technology had been well applied in all aspects of cancer researches [12]. Consequently, through bioinformatics analysis, we tried to find out the cause of $\mathrm{AD}$ and develop a reasonable and feasible early detection program.

The main clinical manifestations of $\mathrm{AD}$ are memory loss and cognitive impairment. And the hippocampus (HIP) is the major functional area of the brain responsible for learning and memory. As the early reliable detection method is still poorly provided, many $\mathrm{AD}$ biomarkers compared the differences of microRNAs or RNA between $\mathrm{AD}$ and healthy elderly people, while cerebrospinal fluid (CSF) is still needed for detection. Blood collecting is more convenient than CSF, and the accuracy of biomarkers could be improved by identifying genes codifferentially expressed in HIP and blood. So we focus on the patients' blood to find genes that are consistent with the changes in HIP. To exploit the pathogenic targets and related biological processes of $\mathrm{AD}$ by the new method, we downloaded mRNA expression profiles GSE1297, GSE5281, GSE18309, and GSE4226 from the Gene Expression Omnibus database to obtain blood and hippocampal samples from $\mathrm{AD}$ patients.

\section{Materials and Methods}

2.1. Microarray Data. Microarray datasets GSE1297, GSE5281, GSE18309, and GSE4226 were downloaded from Gene Expression Omnibus and collected using the following platforms: GPL1211 NIA MGC, Mammalian Genome Collection, GPL570 [HG-U133_Plus_2] Affymetrix Human Genome U133 Plus 2.0 Array, and GPL96 [HG-U133A] Affymetrix Human Genome U133A Array.

2.2. Conversion of Raw Data. The raw data was converted to a recognizable format by GEO2R (https://www.ncbi .nlm.nih.gov/geo/geo2r/). GEO2R performs comparisons on original submitter-supplied processed data tables using the GEOquery package which parses GEO data into $\mathrm{R}$ data structures that can be used by other $\mathrm{R}$ packages and limma (Linear Models for Microarray Analysis) R package from the
Bioconductor project. Each chip group is compared with $\mathrm{AD}$ and NC, respectively.

2.3. Difference Analysis. Difference analysis was performed by GEO2R analysis with $P<0.05$ and $|\log \mathrm{FC}|>1$ as cutoff values for screening differentially expressed genes (DEGs). Enrichment analysis was performed by a Cytoscape [13] plug-in ClueGO [14] with $P<0.05$. ClueGO performed single-cluster analysis and comparison of several clusters. The ClueGO network was created with kappa statistics and reflected the relationships between the terms based on the similarity of their associated genes. Functionally grouped network with terms as nodes was linked based on their kappa score level $(\geq 0.3)$. The intersection of the two sets of DEGS was obtained by VENN diagram. Enrichment results of each chip were term $P$ value, corrected with Bonferroni methods.

\section{Results}

We compared the $\mathrm{AD}$ peripheral blood mononuclear cells (PBMCs) expression dataset (GEO accession GSE4226 and GSE18309) with AD brain tissue expression datasets (GEO accessions GSE1297 and GSE5281) from GEO in the present study. As shown in Table 2, for PBMCs, GSE18309 and GSE4226 included 3 and 14 samples of elderly and 6 and 14 samples of AD patients. For HIPs, GSE1297 and GSE5281 included 9 and 13 elderly samples and 22 and 10 AD samples, respectively. Gene difference analysis found that there were 4958 differential mRNAs in GSE18309, 577 differential mRNAs in GSE4226, 7464 differential mRNAs in GSE5281, and 317 differential mRNAs in GSE1297 compared with $\mathrm{AD}$ patients and healthy elderly. The edger differential gene analysis was performed by using the GEO language analysis tool GEO2R coming with the GEO website. Venn indicated that there were 68 differential mRNAs' expressions in both PBMCs' microarrays (Figure 1(a), Supplemental Table 1) and 154 mRNAs in the two HIP microarrays (Figure 1(b), Supplemental Table 2). In order to obtain the target differential expression gene, we further got two differentially expressed genes, RAB7A and ITGB1, in PBMCs and HIP (Figure 1(c)).

Studying the signaling pathway of gene involvement could help us to understand the role of gene in $\mathrm{AD}$ and further understand the pathological process or cause of AD. ITGB1 was a receptor for IL1 $\beta$ and binding was essential for IL1 $\beta$ signaling [15]. RAB7A was a key regulator in endolysosomal trafficking [16], playing important roles in neurotrophin transport, lipid metabolism, microbial pathogen infection, and survival $[17,18]$. These two genes were also reported as important regulators associated with $\mathrm{AD}$ [19-21]. In order to analyze the possible signaling pathways of RAB7A and ITGB1 in AD, we further enriched and analyzed the four chips. We enriched these genes and analyzed them through the Cytoscape plug-in ClueGo (Figure 2, details shown in Supplemental Figures 1-4). ClueGO integrates GO terms as well as KEGG/BioCarta pathways and creates a functionally organized GO/pathway term network. The network is automatically laid out using the organic layout algorithm supported by Cytoscape. ClueGo also provides the option to calculate mid $\mathrm{P}$ values and doubling for two-sided tests to 
TABLE 1: Current mainstream detection methods of AD.

\begin{tabular}{|c|c|c|c|}
\hline Detection method & Principle & Advantage & Disadvantage \\
\hline MMSE & $\begin{array}{l}\text { The most common scale of } \\
\text { intelligence impairment at present. } \\
\text { Illiteracy } \leq 17 \text { points, primary } \\
\text { school degree } \leq 20 \text { points, middle } \\
\text { school degree } \leq 22 \text { points, college } \\
\text { degree } \leq 23 \text { points, indicates the } \\
\text { impairment of cognitive function. }\end{array}$ & $\begin{array}{l}\text { Low cost, concise content, less time } \\
\text { for measurement, and easy to be } \\
\text { accepted by the elderly }\end{array}$ & $\begin{array}{l}\text { Only screening the patients with } \\
\text { moderate-to-severe symptoms }\end{array}$ \\
\hline $\begin{array}{l}\text { Structural imaging: head } \\
\text { CT (thin scan) and MRI } \\
\text { (coronal) scanning }\end{array}$ & $\begin{array}{l}\text { Diagnostic imaging techniques } \\
\text { show significant atrophy of the } \\
\text { cerebral cortex, especially in the } \\
\text { hippocampus and medial temporal } \\
\text { lobe, supporting the clinical } \\
\text { diagnosis of AD. }\end{array}$ & $\begin{array}{l}\text { Used to exclude other underlying } \\
\text { diseases and to identify specific } \\
\text { imaging findings of } \mathrm{AD}\end{array}$ & $\begin{array}{l}\text { Convenience not as good as MMSE, } \\
\text { expensive cost, hospital equipment } \\
\text { support, low penetration rate }\end{array}$ \\
\hline 18FDG-PET & $\begin{array}{l}\text { It shows the reduction of glucose } \\
\text { metabolism in the dome and } \\
\text { upper/posterior temporal region, } \\
\text { posterior cingulate cortex, and } \\
\text { anterior cranial lobe, revealing } \\
\text { specific abnormal changes in AD. In } \\
\text { the late period of AD, the frontal } \\
\text { lobe metabolism was reduced. }\end{array}$ & $\begin{array}{l}\text { Improving the reliability of } \\
\text { dementia diagnosis and being } \\
\text { suitable for the differential } \\
\text { diagnosis of AD and other dementia }\end{array}$ & $\begin{array}{l}\text { Convenience not as good as MMSE, } \\
\text { expensive cost, specific equipment } \\
\text { support needed, penetration rate }\end{array}$ \\
\hline EEG & $\begin{array}{c}\text { The EEG of AD is characterized by } \\
\text { a decrease in alpha wave, an } \\
\text { increase in theta wave, and a } \\
\text { decrease in the average frequency. }\end{array}$ & $\begin{array}{l}\text { Used for the differential diagnosis of } \\
\mathrm{AD} \text {, providing early evidence of } \\
\text { prion disease or other brain diseases }\end{array}$ & $\begin{array}{c}33 \% \text { of patients with early } \mathrm{AD} \text { are } \\
\text { normal without change in EEG and } \\
\text { low penetration rate }\end{array}$ \\
\hline Biological markers & $\begin{array}{l}\text { Dynamically observing the } \\
\text { progression of the disease by using } \\
\text { proteins or miRNAs in body fluids } \\
\text { such as peripheral blood, } \\
\text { cerebrospinal fluid, and urine } \\
\text { biology as markers to increase the } \\
\text { specificity of the diagnosis. }\end{array}$ & $\begin{array}{l}\text { Noninvasive, simple and } \\
\text { inexpensive, and of low price }\end{array}$ & $\begin{array}{c}\text { Low repeatability, problems with } \\
\text { accuracy and specificity, lack of } \\
\text { standards }\end{array}$ \\
\hline
\end{tabular}

TABLE 2: GEO microarray datasets.

\begin{tabular}{|c|c|c|c|c|}
\hline Chip serial number & Elderly sample & AD sample & Resource & Platform \\
\hline GSE1297 & 9 & 22 & Hippocampus & $\begin{array}{c}\text { GPL1211 NIA MGC, } \\
\text { Mammalian Genome } \\
\text { Collection }\end{array}$ \\
\hline GSE5281 & 13 & 10 & Hippocampus & $\begin{array}{l}\text { GPL1211 NIA MGC, } \\
\text { Mammalian Genome } \\
\text { Collection }\end{array}$ \\
\hline GSE18309 & 3 & 6 & PBMCs & $\begin{array}{c}\text { GPL570 } \\
\text { [HG-U133_Plus_2] } \\
\text { Affymetrix Human } \\
\text { Genome U133 Plus } \\
\text { 2.0 Array } \\
\end{array}$ \\
\hline GSE4226 & 14 & 14 & PBMCs & $\begin{array}{l}\text { GPL96 [HG-U133A] } \\
\text { Affymetrix Human } \\
\text { Genome U133A Array }\end{array}$ \\
\hline
\end{tabular}

deal with discreetness and conservatism effects as suggested (Figure 3, details shown in Supplemental Figures 5-8).

We searched for the enrichment of RAB7A and ITGB1 and select the first five results with the lowest term $P$ value corrected with Bonferroni step-down. In HIP, singleorganism process, positive regulation of cellular and biological process, and cell communication were enriched in
GSE1297, and positive or negative regulation of metabolic process, organelle organization, and signaling regulation were enriched in GSE5281. According to these enrichment results, it was indicated that RAB7A and ITGB1 in HIP were regulatory genes response to metabolism, signaling transduction, and cellular function and communication in AD brain (Table 3, Figures 2(c) and 2(d), and Figures 3(c) 


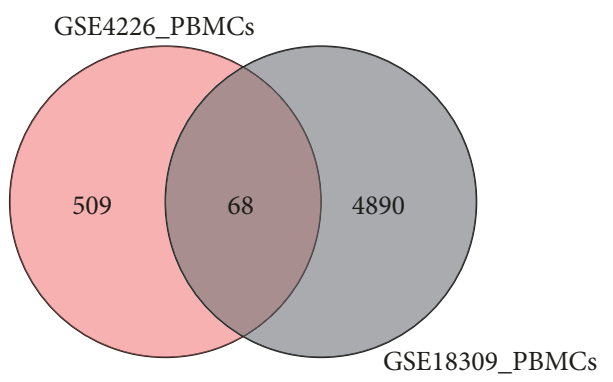

(a)

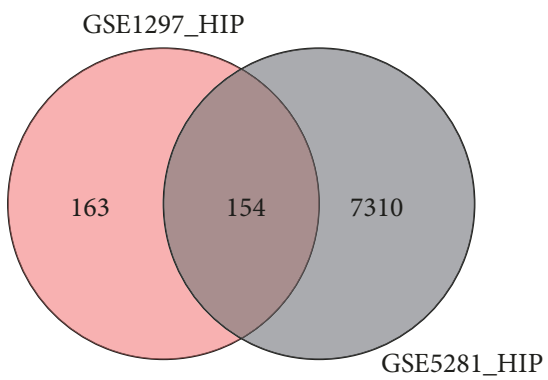

(b)

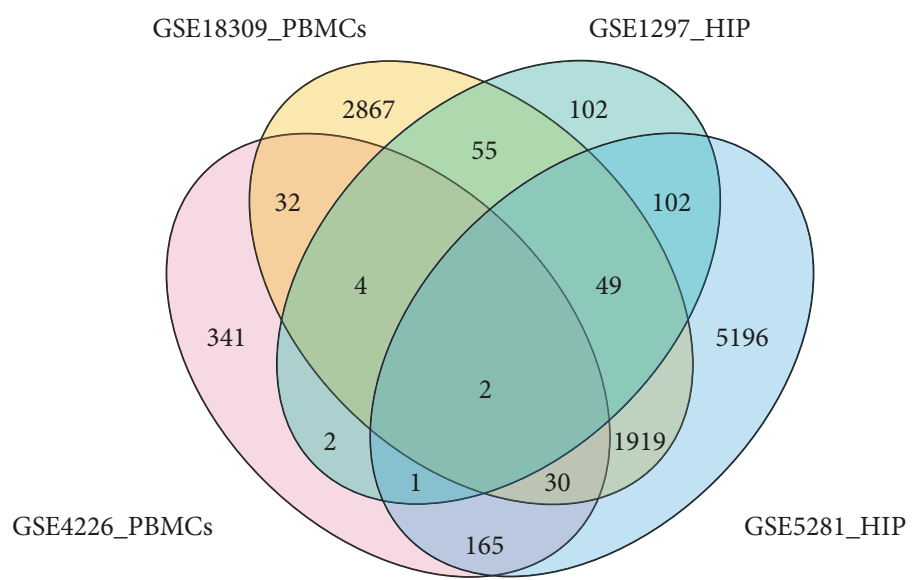

(c)

FIGURE 1: Analysis of differentially expressed genes in AD PBMCs and HIP samples by Venn. (a) Differential mRNAs expression in PBMCs of AD patients. (b) Differential mRNAs expression in HIP of AD patients. (c) Coexpression of differentially expressed mRNAs in the PBMCs and HIP of AD patients.

and 3(d)). Furthermore, in PBMCs, symbiosis (encompassing mutualism through parasitism), viral process and life cycle, catabolic process, and cellular protein localization were enriched in GSE4226; immune response, locomotion, regulation of localization, leukocyte activation, and signaling were enriched in GSE18309 (Table 3 , Figures 2(a) and 2(b), and Figures 3(a) and 3(b)). It was suggested that immune response and inflammatory changes to the microorganism or stimuli were the main changes in AD PBMCs.

As far as the two genes are concerned, the involving functions in PBMCs were not so similar to those in HIP. However, we found that they had close relationship with each other. Activation of immune response releases many proinflammatory cytokines. Long-term activation of the immune system in AD exacerbated neuroinflammation and contributes to neurodegeneration [22]. Recruitment of peripheral macrophages into the brain led to deterioration of $\mathrm{AD}$ pathology, which might aggravate the effects of persistent inflammation and AD pathology [23]. Also, neurodegeneration further stimulated the immune system to become a malignant cycle and caused systemic effects [24]. Therefore, it meant that the changes of RAB7A and ITGB1 in PBMCs could be premonitory signs of AD which would lead to some consequent biological changes in HIP and finally to cognitive problems.

\section{Discussion}

$\mathrm{AD}$ was an irreversible progressive neurodegenerative disease that can only be controlled by early intervention for early prevention and treatment. To date, there are mainly MMSE, medical imaging, or biomarkers to detect $\mathrm{AD}$, but the early prediction results were not satisfactory, suggesting that new early biomarkers needed to be obtained, and effective and efficient detection schemes should be developed.

At present, bioinformatics prediction and computer technology had been well applied in all aspects of biomedicine research [25]. However, there are not many bioinformatics analyses in Alzheimer's disease, and the current research on Alzheimer's disease was mainly concentrated in single site, which lacks multiple sites for joint research. Chip analysis and screening allow researchers to obtain many differential genes. The expressed data provided a novel approach to study the detection and targeted therapy of $\mathrm{AD}$ or to exploit new biomarker.

By comparing the hippocampus and peripheral blood of $\mathrm{AD}$ patients with normal elderly group, 154 differentially expressed genes in hippocampus and 68 differentially expressed genes in peripheral blood were obtained. We found that ITGB1 and RAB7A were differentially expressed in HIP and $\mathrm{PBMCs}$ of $\mathrm{AD}$ patients by Venn diagram compared 


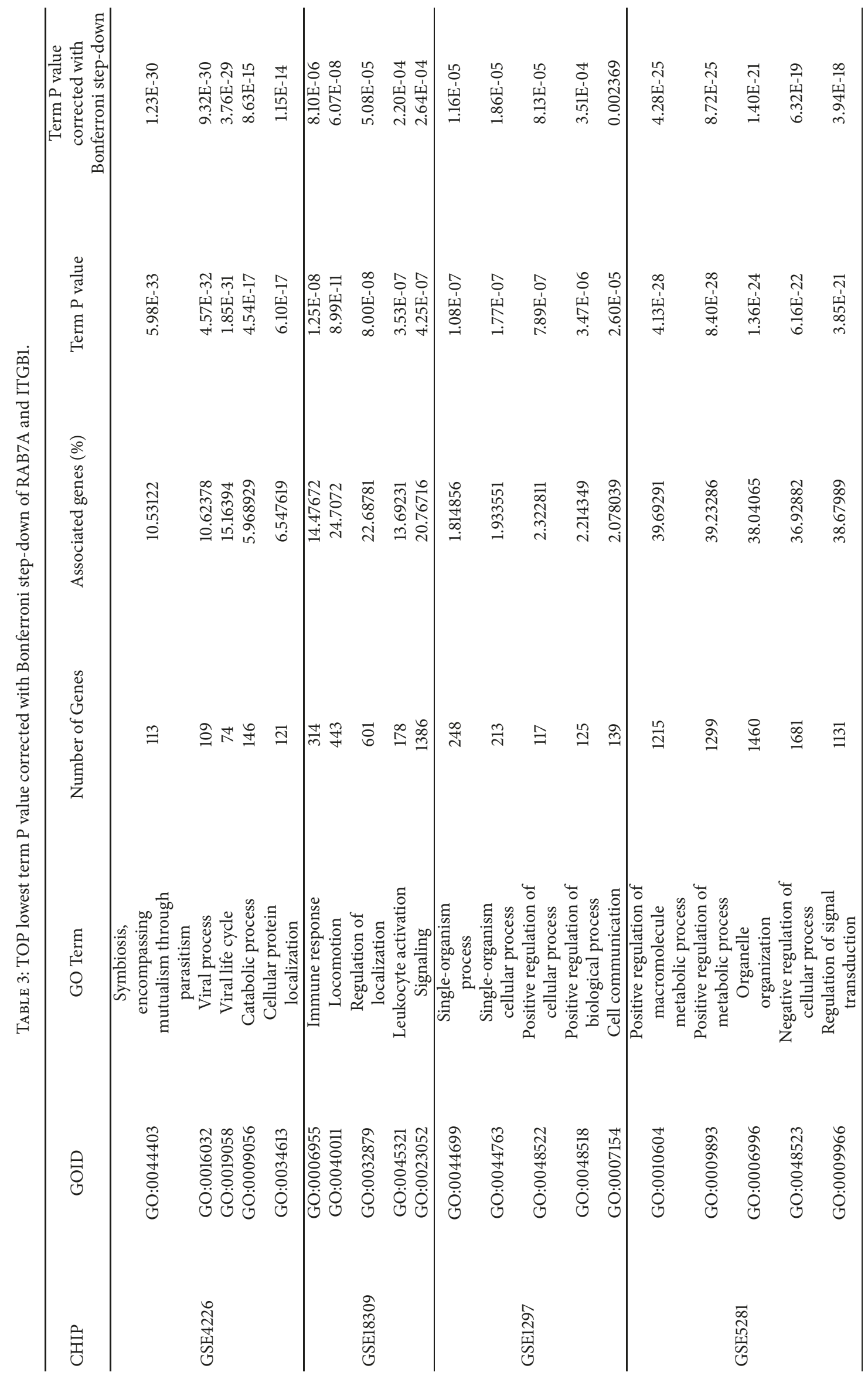




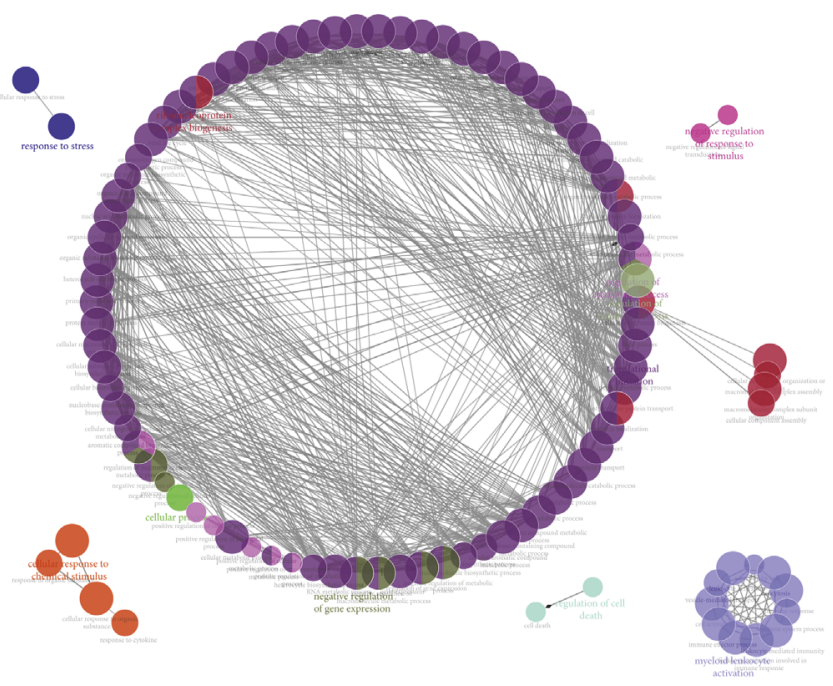

(a)

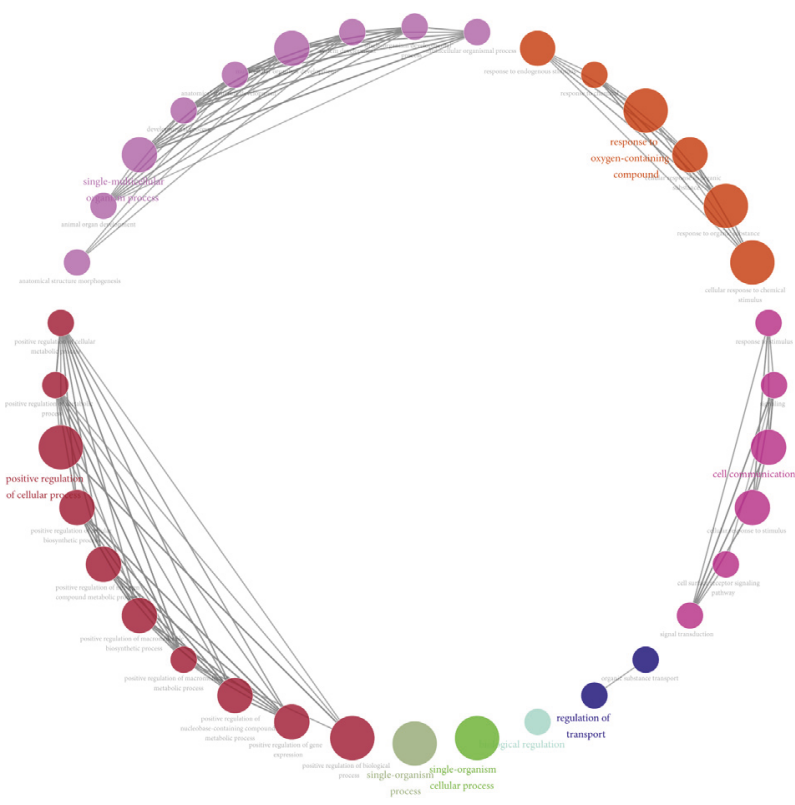

(c)

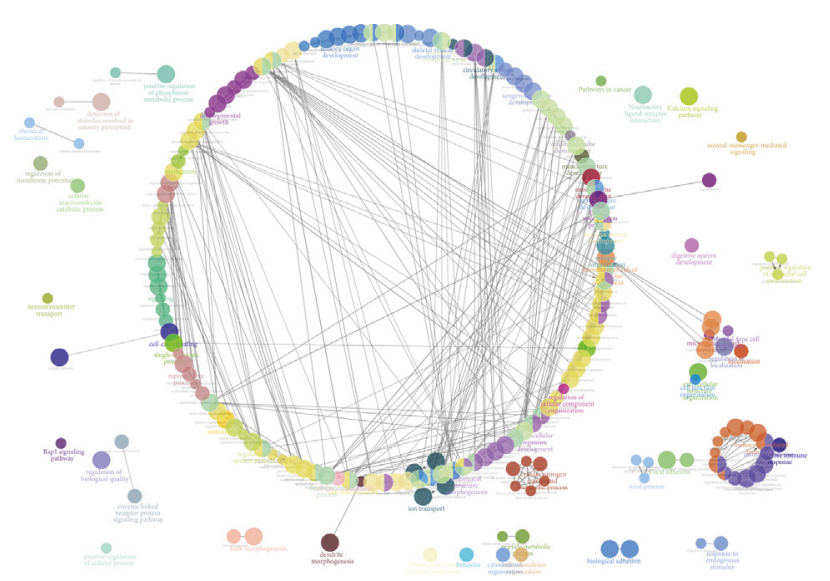

(b)

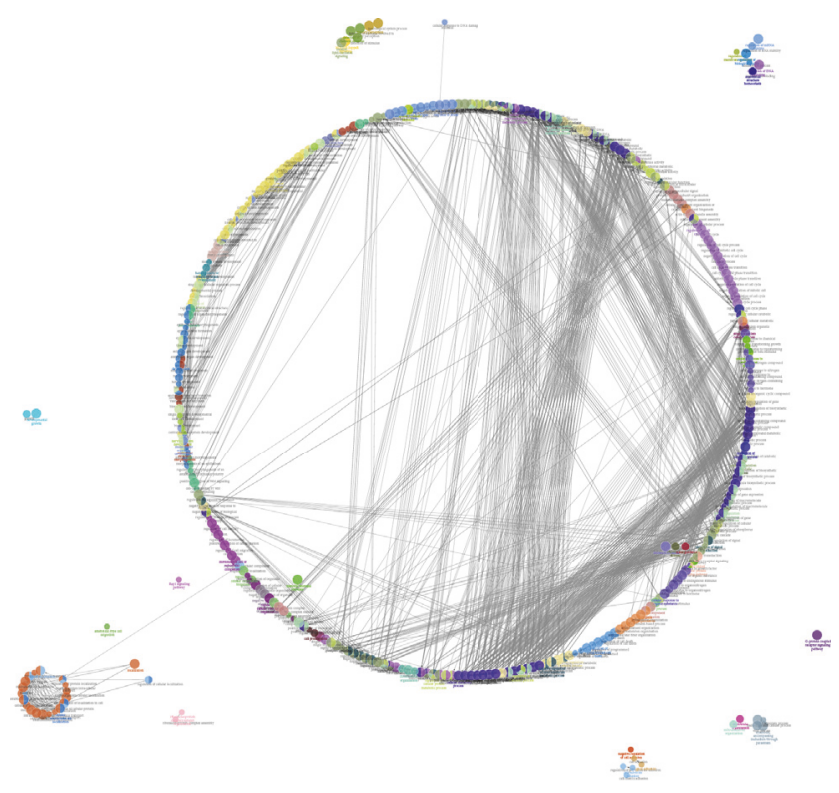

(d)

Figure 2: Enrichment network analysis of GSE4226 (a), GSE18309 (b), GSE1297 (c), and GSE5281 in the PBMCs or HIP of AD patients. To further capture the relationship between terms, a subset of enriched terms was selected and drawn as a network graph. Their representative enriched terms were colored by different color. The line showed the relative of spot that was connected. Functionally grouped network with terms as nodes was linked based on their kappa score level $(\geq 0.3)$, where only the label of the most significant term per group is shown in color.

with healthy elderly people. RAB7A was involved in tau secretion in the brain and played an important role in the transport of neurotrophic factors in animal and cell experiments [21, 26, 27]. Besides, RAB7A plays a key role in neurotrophin transport and lipid metabolism in neuronal axons [17]. At the same time, RAB7A also plays an important role in the infection and survival of microbial pathogens [18]. The other screened gene was ITGB1, which is a receptor for IL1 $\beta$ and binding is essential for IL1 $\beta$ signaling [15]. As we know, IL1 $\beta$ plays a key role in the occurrence and development of $\mathrm{AD}$ [28], so ITGB1 also contributed to AD progression. It was reported that ITGB1 interacted with APP to regulate axons outward growth and adhesion and affects cell migration, which was supported by many literatures [21, 26, 27]. Moreover, ITGB1 plays an important role in the regulation of immune response [29], consistent with our results. Even though their biological function enrichment in PBMCs was not similar to those in HIP, there was a close relationship between the inflammatory and immune changes in peripheral blood and the signaling regulation in HIP. The immune system secretes proinflammatory cytokines, leading to the emergence of responses [30]. Inflammation plays an 


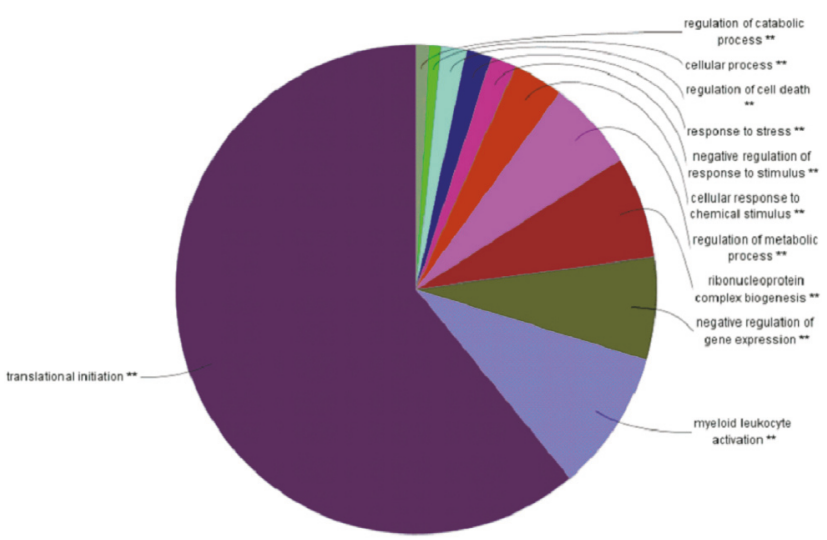

(a)

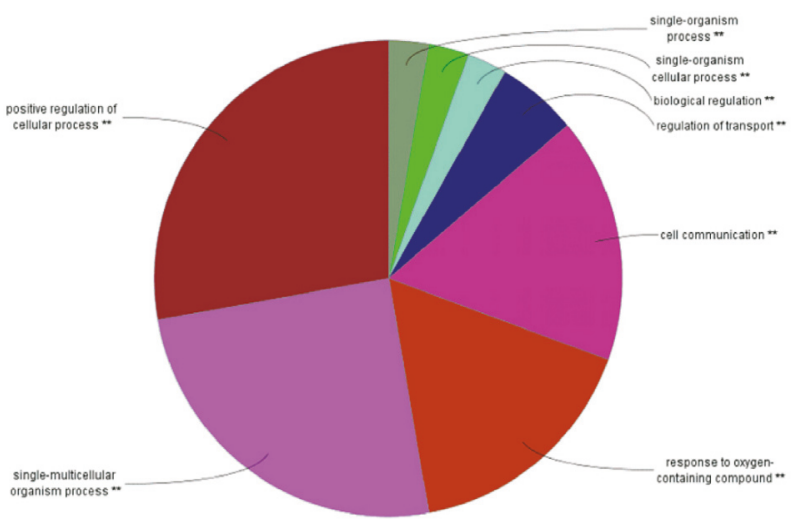

(c)

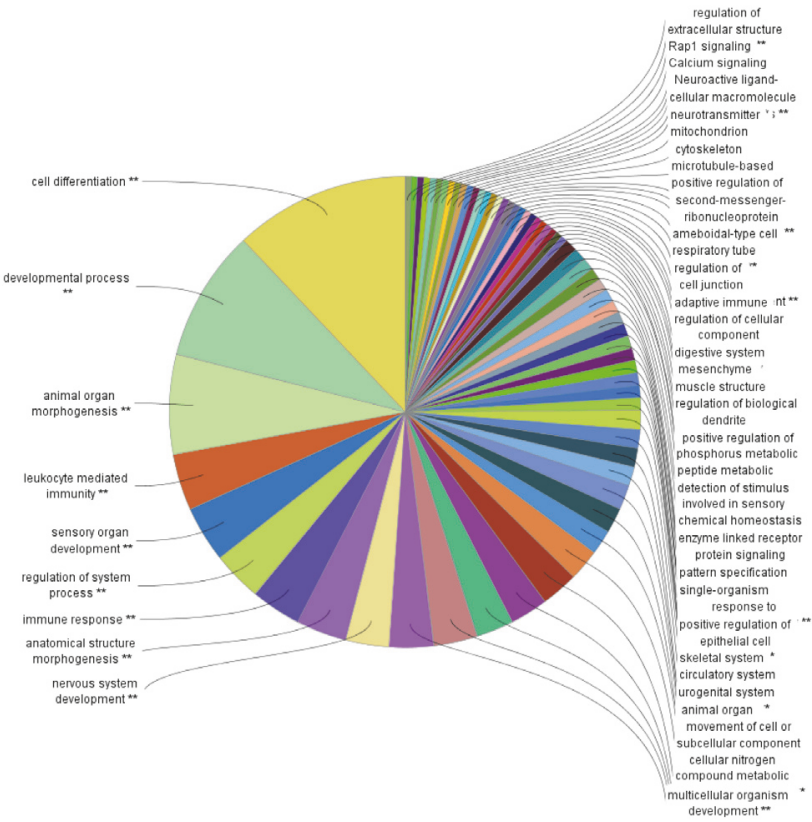

(b)

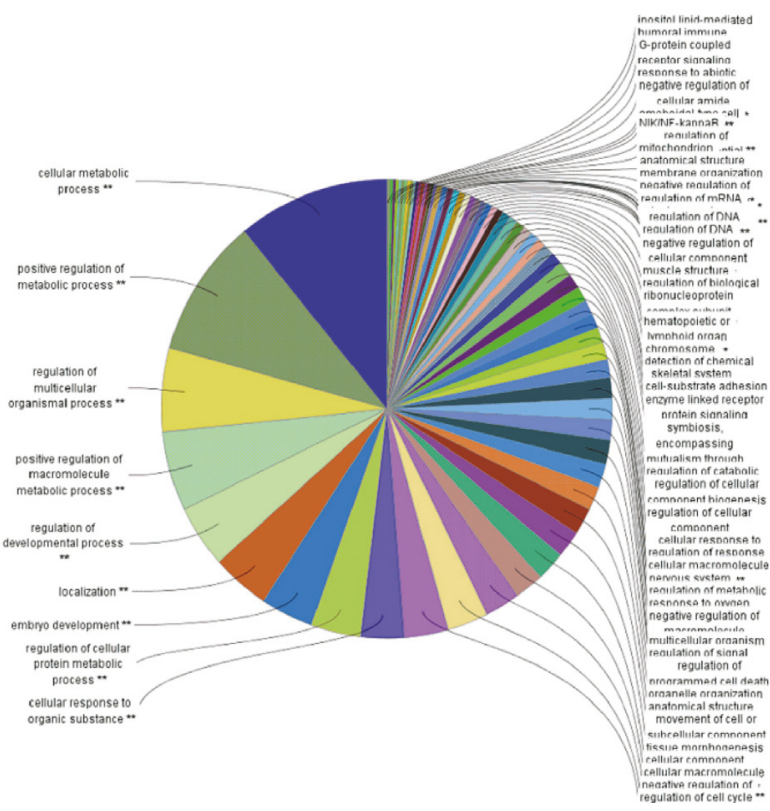

(d)

FIGURE 3: Overview chart with functional groups of each chip with term P value corrected. (a) GSE4226. (b) GSE18309. (c) GSE1297. (d) GSE5281.

important role in the pathogenesis of $\mathrm{AD}$. Current studies have shown that there are strong inflammatory reactions mediated by microglia and astrocytes in $\mathrm{AD}$ patients' brain [31]. An accompanying change of metabolism, signaling, and biological process in HIP followed after the immune and inflammatory changes in PBMCs.

According to our screening results, ITGB1 and RAB7A were potential biomarkers for the prediction and diagnosis of $\mathrm{AD}$. Therefore, this meant that the patients could be diagnosed by detecting peripheral blood, without brain biopsy or cerebrospinal fluid detection. However, the patient's blood samples can be detected by PCR to determine the condition; the threshold for the diagnosis of $\mathrm{AD}$ needs to be determined by experiments. In addition to detecting the expression of ITGB1 and RAB7A for AD screening, we recommended that the routine blood of the elderly was tested for 2-3 years at a frequency of 6 months or 1 year. If long-term immune system abnormalities are found in the 
elderly, the patients need to be treated or intervened as earlier as possible. However, the cut-off value should be set in normal physiological range of normal healthy population. This would be conducive to hospital implementation and diagnosis. Although inflammation did not necessarily lead to $\mathrm{AD}, \mathrm{AD}$ patients usually have a long-term inflammatory response.

We believed that inflammation is a signal of early pathological changes in AD. Severe inflammatory reactions in middle-aged people had been reported to increase the risk of $\mathrm{AD}$ [32]. Therefore, we speculated that the risk of $\mathrm{AD}$ in elderly patients with long-term immune system abnormalities is much higher than that in normal elderly people. The treatment or intervention of inflammatory reaction could effectively delay or delay the occurrence and development of $\mathrm{AD}$. On the other hand, elderly patients with long-term immune system abnormalities also needed to be treated. For potential high-risk patients, hospital should treat or intervene in the inflammation of patients before the clinical manifestation emerging in order to achieve the effects of treatment and prevention of early $\mathrm{AD}$.

\section{Conclusions}

ITGB1 and RAB7A mRNA expressions were both changed in hippocampus and PBMCs, highly suggested being used as an $\mathrm{AD}$ biomarker with $\mathrm{AD}$. Also, according to the results of this analysis, it is indicated that we can test the blood routine of the elderly for 2-3 years at a frequency of 6 months or one year. When a patient continuously detects the inflammatory manifestations, they are indicated as a potentially high-risk $\mathrm{AD}$ patient for $\mathrm{AD}$ prevention.

\section{Data Availability}

The data used to support the findings of this study are available from the corresponding author upon request.

\section{Conflicts of Interest}

All the authors declare no potential conflicts of interest.

\section{Acknowledgments}

This work was supported by the National Natural Science Foundation of China (nos. 81501098, 81603112, and 81703519), Key R\&D Plan Guidance Project of Liaoning Province (2018225089), "Double Hundred Program" for Shenyang Scientific and Technological Innovation Projects (100040), the Drug Innovation Major Project (2018ZX09711001-008006), Program for Liaoning Innovation Research Team in University (no. LT2014016), Key Laboratory Foundation from Shenyang S\&T Projects (F16-094-1-00), and Liaoning Province Scientific Research Foundation (2014226033).

\section{Supplementary Materials}

Supplemental Table 1 is the list of 68 differential mRNAs' expressions in both PBMCs' microarrays. Supplemental Table 2 is the list of 154 mRNAs in the two HIP microarrays. Supplemental Figures 1-4 correspond to Figures 2(a)-2(d). Supplemental Figures 5-8 correspond to Figures 3(a)-3(d). (Supplementary Materials)

\section{References}

[1] A. Alzheimer, "Über eine eigenartige Erkrankung der Hirnrinde. Allgemeine zeitschrift fur psychiatrie und psychischgerichtlich," Medizin, vol. 64, pp. 146-148, 1907.

[2] N. K. Isaev, E. V. Stelmashook, E. E. Genrikhs, M. V. Oborina, M. R. Kapkaeva, and V. P. Skulachev, "Alzheimer's disease: an exacerbation of senile phenoptosis," Biochemistry (Moscow), vol. 80, no. 12, pp. 1578-1581, 2015.

[3] M. J. Prince, "World Alzheimer Report 2015: The Global Impact of Dementia: an Analysis of Prevalence, Incidence, Cost and Trends," 2015.

[4] J. H. Barnett, L. Lewis, A. D. Blackwell, and M. Taylor, "Early intervention in Alzheimer's disease: a health economic study of the effects of diagnostic timing," BMC Neurology, vol. 14, no. 101, 2014.

[5] A. Kumar, A. Singh, and Ekavali, "A review on Alzheimer's disease pathophysiology and its management: an update," Pharmacological Reports, vol. 67, no. 2, pp. 195-203, 2015.

[6] M. M. Dorostkar, C. Zou, L. Blazquez-Llorca, and J. Herms, "Analyzing dendritic spine pathology in Alzheimer's disease: problems and opportunities," Acta Neuropathologica, vol. 130, no. 1, pp. 1-19, 2015.

[7] C. S. Atwood and R. L. Bowen, "A unified hypothesis of earlyand late-onset alzheimer's disease pathogenesis," Journal of Alzheimer's Disease, vol. 47, no. 1, pp. 33-47, 2015.

[8] CADTH Rapid Response Reports, Brain Electrical Activity Mapping for Diagnosing Psychiatric Disorders: A Review of the Clinical Evidence, Canadian Agency for Drugs and Technologies in Health, Ottawa (ON), Nov, 2014.

[9] C. Marcus, E. Mena, and R. M. Subramaniam, "Brain PET in the diagnosis of Alzheimer's disease," Clinical Nuclear Medicine, vol. 39, no. 10, pp. e413-e426, 2014.

[10] S. Bhat, U. R. Acharya, N. Dadmehr, and H. Adeli, "Clinical neurophysiological and automated EEG-based diagnosis of the alzheimer's disease," European Neurology, vol. 74, no. 3-4, pp. 202-210, 2015.

[11] M. S. Fiandaca, M. E. Mapstone, A. K. Cheema, and H. J. Federoff, "The critical need for defining preclinical biomarkers in Alzheimer's disease," Alzheimer's \& Dementia, vol. 10, Suppl 3, pp. S196-S212, 2014.

[12] J. K. Banwait and D. R. Bastola, "Contribution of bioinformatics prediction in microRNA-based cancer therapeutics," Advanced Drug Delivery Reviews, vol. 81, pp. 94-103, 2015.

[13] P. Shannon, A. Markiel, O. Ozier et al., "Cytoscape: a software environment for integrated models of biomolecular interaction networks," Genome Research, vol. 13, no. 11, pp. 2498-2504, 2003.

[14] G. Bindea, B. Mlecnik, H. Hackl et al., "ClueGO: a Cytoscape plug-in to decipher functionally grouped gene ontology and pathway annotation networks," Bioinformatics, vol. 25, no. 8, pp. 1091-1093, 2009. 
[15] Y. K. Takada, J. Yu, M. Fujita, J. Saegusa, C. Wu, and Y. Takada, "Direct binding to integrins and loss of disulfide linkage in interleukin-1 $\beta$ (IL-1 $\beta$ ) are involved in the agonistic action of IL-1 $\beta$," The Journal of Biological Chemistry, vol. 292, no. 49, pp. 20067-20075, 2017.

[16] G. Cantalupo, P. Alifano, V. Roberti, C. B. Bruni, and C. Bucci, "Rab-interacting lysosomal protein (RILP): The Rab7 effector required for transport to lysosomes," EMBO Journal, vol. 20, no. 4, pp. 683-693, 2001.

[17] The UniProt Consortium, "Uniprot: the universal protein knowledgebase," Nucleic Acids Research, vol. 45, no. D1, pp. D158-D169, 2017.

[18] S. Seto, K. Tsujimura, and Y. Koide, "Rab GTpases regulating phagosome maturation are differentially recruited to mycobacterial phagosomes," Traffic, vol. 12, no. 4, pp. 407-420, 2011.

[19] K. Tominaga, T. Yoshimoto, K. Torigoe et al., "IL-12 synergizes with IL-18 or IL-1beta for IFN-gamma production from human T cells," International Immunology, vol. 12, no. 2, pp. 151-160, 2000.

[20] T. Chitnis and H. L. Weiner, "CNS inflammation and neurodegeneration," The Journal of Clinical Investigation, vol. 127, no. 10, pp. 3577-3587, 2017.

[21] L. Rodriguez, N.-V. Mohamed, A. Desjardins, R. Lippé, E. A. Fon, and N. Leclerc, "Rab7A regulates tau secretion," Journal of Neurochemistry, vol. 141, no. 4, pp. 592-605, 2017.

[22] J. W. Kinney, S. M. Bemiller, A. S. Murtishaw, A. M. Leisgang, A. M. Salazar, and B. T. Lamb, "Inflammation as a central mechanism in Alzheimer's disease," Alzheimer's \& Dementia: Translational Research \& Clinical Interventions, vol. 4, pp. 575590, 2018.

[23] T. R. Jay, C. M. Miller, P. J. Cheng et al., “TREM2 deficiency eliminates TREM2+ inflammatory macrophages and ameliorates pathology in Alzheimer's disease mouse models," The Journal of Experimental Medicine, vol. 212, no. 3, pp. 287-295, 2015.

[24] K. Bisht, K. Sharma, and M.-È. Tremblay, "Chronic stress as a risk factor for Alzheimer's disease: Roles of microgliamediated synaptic remodeling, inflammation, and oxidative stress," Neurobiology of Stress, vol. 9, pp. 9-21, 2018.

[25] Q. Zou, D. Mrozek, Q. Ma et al., "Scalable data mining algorithms in computational biology and biomedicine," BioMed Research International, vol. 2017, Article ID 5652041, 3 pages, 2017.

[26] S. Zafar, N. Younas, S. Correia et al., "Strain-specific altered regulatory response of rab7a and tau in creutzfeldt-jakob disease and alzheimer's disease," Molecular Neurobiology, vol. 54, no. 1, pp. 697-709, 2017.

[27] L. J. Sosa, J. Bergman, A. Estrada-Bernal, T. J. Glorioso, J. M. Kittelson, and K. H. Pfenninger, "Amyloid precursor protein is an autonomous growth cone adhesion molecule engaged in contact guidance," PLoS ONE, vol. 8, no. 5, Article ID e64521, 2013.

[28] C. S. White, C. B. Lawrence, D. Brough, and J. Rivers-Auty, "Inflammasomes as therapeutic targets for Alzheimer's disease," Brain Pathology, vol. 27, no. 2, pp. 223-234, 2017.

[29] Z. Li, Z. Xiong, L. C. Manor, H. Cao, and T. Li, "Integrative computational evaluation of genetic markers for Alzheimer's disease," Saudi Journal of Biological Sciences, vol. 25, no. 5, pp. 996-1002, 2018.

[30] S. S. Chavan, V. A. Pavlov, and K. J. Tracey, "Mechanisms and therapeutic relevance of neuro-immune communication," Immunity, vol. 46, no. 6, pp. 927-942, 2017.
[31] I. Olsen and S. K. Singhrao, "Inflammasome involvement in Alzheimer's disease," Journal of Alzheimer's Disease, vol. 54, no. 1, pp. 45-53, 2016.

[32] C. L. Sutphen, M. S. Jasielec, A. R. Shah et al., "Longitudinal cerebrospinal fluid biomarker changes in preclinical alzheimer disease during middle age," JAMA Neurology, vol. 72, no. 9, pp. 1029-1042, 2015 


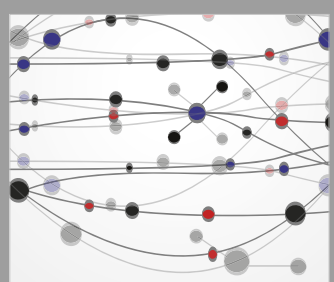

The Scientific World Journal
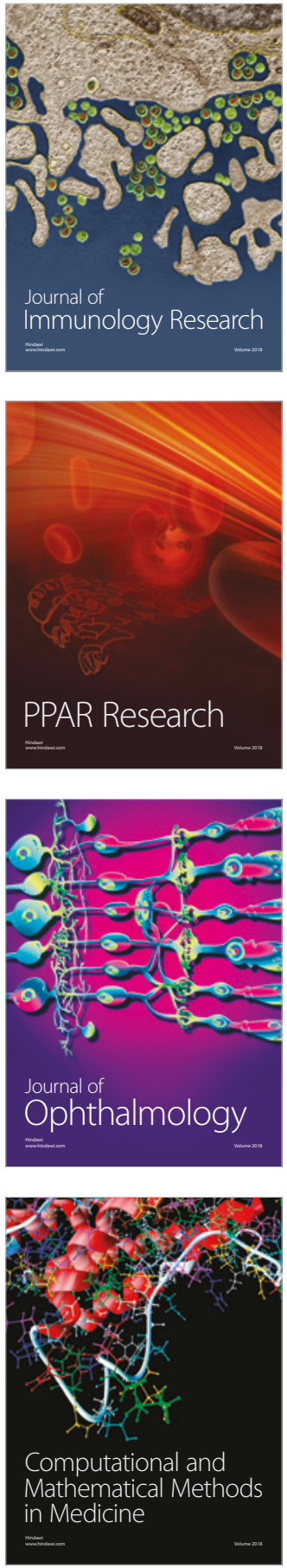

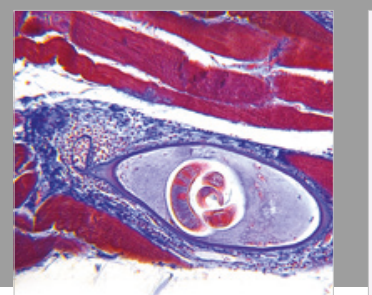

Gastroenterology Research and Practice

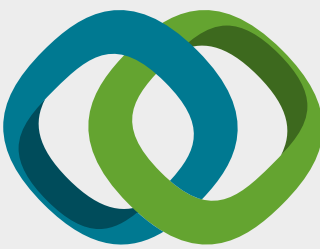

\section{Hindawi}

Submit your manuscripts at

www.hindawi.com
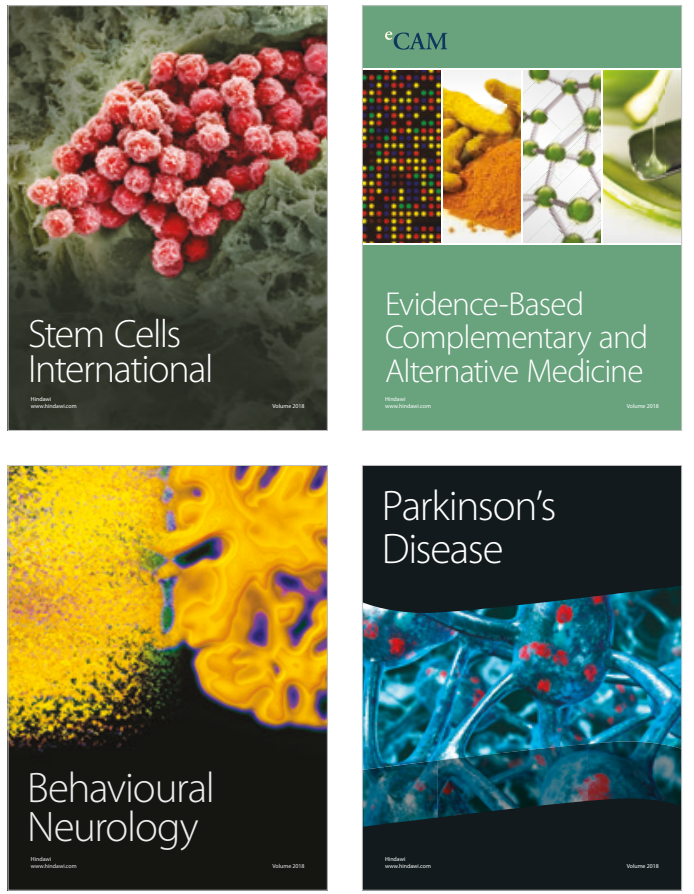

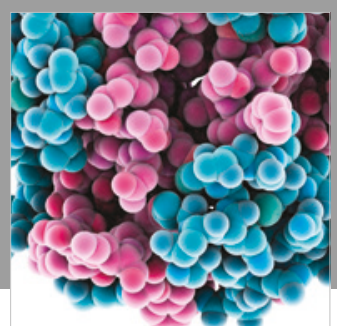

ournal of

Diabetes Research

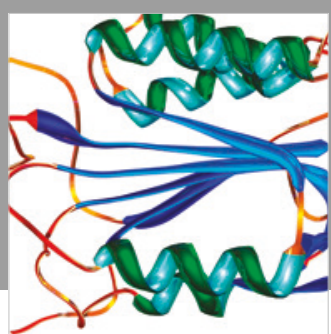

Disease Markers
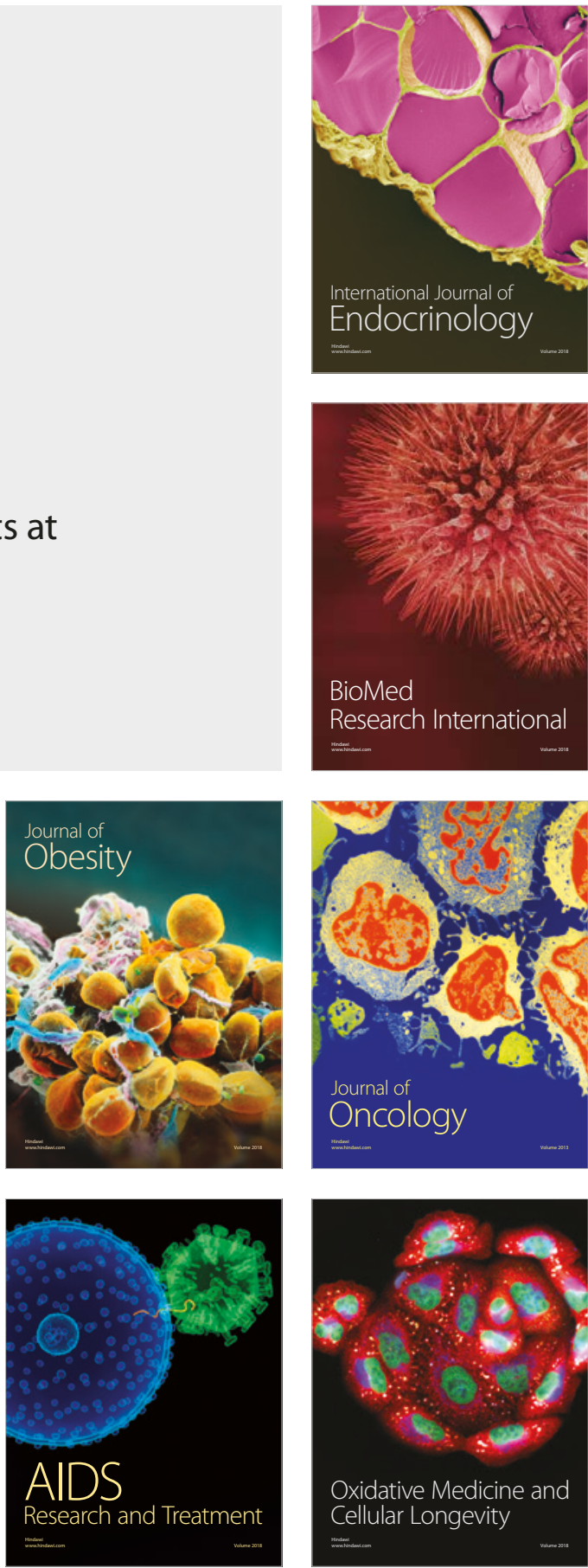